\title{
Intrinsic Helical Twist and Chirality in Ultrathin Tellurium Nanowires
}

Alejandra Londono-Calderon ${ }^{1}$, Darrick Williams ${ }^{2}$, Benjamin Savitzky ${ }^{3}$, Colin Ophus ${ }^{4}$, Sijie Ma ${ }^{5}$, Hanyu $\mathrm{Zhu}^{5}$, Matthew Schneider ${ }^{2}$ and Michael Pettes ${ }^{2}$

${ }^{1}$ Los Alamos National Laboratory, Los Alamos, New Mexico, United States, ${ }^{2}$ Los Alamos National Laboratory, United States, ${ }^{3}$ Lawrence Berkeley National Laboratory, Berkeley, California, United States,

${ }^{4}$ National Center for Electron Microscopy, Berkeley, California, United States, ${ }^{5}$ Rice University, United States

Monoelemental nanomaterials with lattice anisotropy are emerging candidates for nano-electronics devices. Exciting properties emerge with non-centrosymmetric crystallinity such as anisotropic thermal conductivity, spin texture, and gyrotropic effects. Nanostructured trigonal tellurium (t-Te) is an air-stable promising candidate for new nanotechnologies due to its high hole mobility, high current density, and predicted larger electronic bandgap compared to the bulk, making it a useful channel material for extremely scaled FETs.1 The inherent one-dimensional (1D) crystalline structure of Te consists of covalently bonded atoms in a helical chain arrangement along the z-axis with right- or left-handed (P3121or P3221 space group respectively) chirality. Handedness also provides possible optics and plasmonic applications, making ultrathin (sub $10 \mathrm{~nm}$ ) Te a versatile material. The preferentially driven growth of Te along the helical chain axis promotes the formation of 1D nanostructures such as nanoribbons, nanotubes, and nanowires (NWs). Nanoscaled chirality provides a means to tune specific responses as the properties become orientation-dependent; however, atomic chirality is rarely expressed at the nanoscale. Several methodologies have been implemented in various types of NWs to express chirality beyond the unit cell by the spontaneous/controlled formation of screw dislocations, 2 or by directing the handedness with chiral biomolecules3 to promote a periodic helical twist along the axis of the NW. In this presentation, helicity and chirality of ultrathin Te NWs were identified using fourdimensional scanning transmission electron microscopy (4D-STEM) and second harmonic generation circular dichroism (SHG-CD). The subtle changes in orientation along the axis of the nanowire show a previously undetected helical twist along the length axis with enough periodicity to produce nanoscaled ordered chirality. Lattice rotation along the NW's axis was identified with a custom-made postprocessing automated crystal orientation mapping (ACOM) script by template matching the nanobeam electron diffraction (NBED) patterns in the 4D-STEM dataset with a library of possible orientations.4 Additional analysis using a classification method of common NBED patterns into classes provides complementary information by increasing the signal-to-noise ratio and compensate for artifacts related to weak scattering of the ultrathin NWs. Orientation changes were more reliably detected in the averaged classes over individual NBED patterns. Evidence of reciprocal lattice rotation from the [2-1-10] to the [10-10] zone axis about the [0001] direction was observed with an approximate twist rate of $0.2-0.3^{\circ} \mathrm{nm}-1$. Circularpolarization dependent SHG response confirms the net chirality of randomly-ordered bundles of twisted ultrathin Te NWs; by contrast, as-grown 2D Te nanosheets do not exhibit any net SHG-CD above the uncertainty level.5

\section{References}

1. Kramer, A.; Van de Put, M. L.; Hinkle, C. L.; Vandenberghe, W. G., Tellurium as a successor of silicon for extremely scaled nanowires: A first-principles study. npj 2D Materials and Applications 2020, 4 (1), 10-1-8. 
2. Sung, B.; de la Cotte, A.; Grelet, E., Chirality-controlled crystallization via screw dislocations. Nature Communications 2018, 9 (1), 1405-1-7.

3. Ben-Moshe, A.; Wolf, S. G.; Sadan, M. B.; Houben, L.; Fan, Z.; Govorov, A. O.; Markovich, G., Enantioselective control of lattice and shape chirality in inorganic nanostructures using chiral biomolecules. Nat. Commun. 2014, 5 (1), 4302-1-9.

4. Hong, X.; Zeltmann, S.; Savitzky, B.; DaCosta, L. R.; Mueller, A.; Minor, A. M.; Bustillo, K.; Ophus, C., Multibeam Electron Diffraction. arXiv 2020, arXiv:2009.09134.

5. This work was supported by the LDRD program of Los Alamos National Laboratory under project number 20190516ECR and the NSEC projects IMS RR19PETT and IMS RR21PETT, and was performed at the Center for Integrated Nanotechnologies at Los Alamos National Laboratory. Los Alamos National Laboratory is managed by Triad National Security, LLC for the U.S. Department of Energy's NNSA, under contract 89233218CNA000001. 